\section{Sex and the origin of genetic exchanges}

\author{
Thierry Lodé \\ Université de Rennes 1, Campus de \\ Beaulieu, France
}

\begin{abstract}
Sex refers to any biological process selected for genetic exchange but the reason for why sex is so common in Eukaryotes continues to resist understanding. Bacteria appear to adapt and proliferate despite the fact that they lack genes for sex. To understand why sex has evolved, we must answer two crucial questions: i) what are the key differences between eukaryotes and bacteria and ii) why do some eukaryotes use asexual reproduction. Recently, new ideas about the evolution of sex are being proposed which point out that sex and recombination could be the result of very primitive interactions.
\end{abstract}

\section{Introduction}

Although sex has been found to be the most common mode of reproduction among eukaryotes, the hypothetical advantages of sexual reproduction remain a major unsolved issue in evolution.1,2 Sexual reproduction certainly requires an important mobilization of energy at each stage from the development of gametes to courtship. The evolutionary advantage of sexual reproduction has been attributed both to adaptation to changing environments and to the reduction of deleterious mutations. ${ }^{3}$ However, the potential longterm benefits to the species may be insufficient to explain the origin and short-term persistence of sexual reproduction in individuals. 4 Therefore, numerous authors have argued for pluralistic approaches mixing mutation-recombination models. ${ }^{5-7}$

Nevertheless, sex has a significant evolutionary cost, called the two-fold cost of sex. ${ }^{8,9}$ While asexual reproduction allows an exponential increase in the number of offspring in each generation, the cost of maintaining males and the production of gametes reduce the fitness of sexual populations. Each breeder transmits only half of its own genes to each of the offspring. Primitive eukaryotes have been known for two billion years. ${ }^{10}$ Consequently, although sex and meiosis appeared a long time ago, ${ }^{11,12}$ surprisingly, sex results in a very costly mechanism that wastes many specialized cells as well as considerable energy.

Although most of primitive cellular interac- tions are mainly antagonistic (phagocytosis, parasitism, etc...), sexual reproduction is a stable efficient interaction widespread in eukaryotes. ${ }^{13}$ Therefore, the persistence of sex over more than two billion years in many diverse lineages requires a unified explanation. In fact, to understand why sex has evolved, we must answer two crucial questions: i, what are the key differences between eukaryotes and bacteria in which sexual reproduction does not occur, ${ }^{14}$ and ii, why do some eukaryotes appear as exceptions in developing asexual reproduction, such as parthenogenesis or scissiparity.

\section{Genetic exchanges}

Sex refers to any biological process selected for genetic exchange ${ }^{15}$ and is characterized by the alternation of a haploid stage that follows meiosis with a diploid stage following recombination. Although the evolutionary origin of recombination is often described as the origin of sex, there is a distinction between recombination and sex. Recombination is a chemical process rooted in nucleic-acid strand exchange, while sex appears as a biological process rooted in cellular events. ${ }^{16-18}$ While sex (i.e. syngamy, nuclear fusion and meiosis) is found only in eukaryotes, prokaryotes also experience recombination either through direct genetic transfer (bacterial conjugation) or through uptake of exogenous DNA from the environment (transformation, transduction) suggesting that the mechanisms of recombination could be universal. ${ }^{19}$ Nevertheless, these bacterial genetic transfers remain both incomplete, depending on the duration of conjugation, and non-reciprocal, from a donor to a recipient cell.

In contrast, in eukaryotes, sexual reproduction involves a transfer of genetic material through almost complete recombination and meiosis. Although Rec proteins are implicated in bacterial DNA repair mechanisms, $13-15,20$ bacteria can often transmit genetic material in the form of a plasmid in a recipient bacterium but with no recombination of the main chromosome (Figure 1), and there is neither true conjugation nor meiosis among prokaryotes. In rare cases, fragments of chromosomal DNA can migrate into a recipient bacterium. Nevertheless, in most situations, the donor bacterium seems to transfer host genes only by accident. Therefore, this transfer of genetic material does not appear to constitute a recombination per se and it can be said that bacteria do not practice sex. ${ }^{20}$

Further, prokaryotes lack a nuclear envelope including lamina and lack the features required for a true mitotic process. 21,22

While bacteria carry genetic material in the form of a unique chromosome and are usually
Correspondence: Thierry Lodé, UMR CNRS 6552 ETHOS, Université de Rennes 1, Campus de Beaulieu, France change campus de beaulieu into UMR CNRS 6552. France.

E-mail: thierry.lode@univ-angers.fr

Key words: asexual reproduction, eukaryotes, horizontal gene transfer, meiosis, sex origin, libertine bubble theory

Acknowledgments: I am grateful to anonymous referees for helpful comments on the manuscript. I apologize to all colleagues whose work I may have failed to cite in this article.

Received for publication: 1 September 2011. Revision received: 20 December 2011. Accepted for publication: 22 December 2011.

This work is licensed under a Creative Commons Attribution NonCommercial 3.0 License (CC BYNC 3.0).

(O) Copyright T. Lodé, 2012

Licensee PAGEPress, Italy

Trends in Evolutionary Biology 2012; 4:e1 doi:10.4081/eb.2012.e1

haploid, the size of eukaryotic genomes is very much larger, and most species are diploid, sometimes polyploid, only gametes are haploid. Furthermore, histones and histonelike proteins are found in the nucleus of eukaryotic cells, but not in bacteria. Eukaryotic chromosomes are retained in a mass of chromatin, and it could be assumed that three major points - meiosis, diploidy and packaging of genetic material in a nuclear envelope - are the key elements for the evolutionary emergence of sex.

Evolutionary scenarios on the origin of sex could explain why sex is the favoured mode of reproduction in eukaryotes. It may be expected that genetic exchanges are already present in archaic phases of proto-cell organization. The evolution of sex can be described as a type of gene exchange that occurs independently from reproduction. ${ }^{23}$ For instance, conjugation in protists is an exchange of genetic material through a mechanism similar to horizontal gene transfer. ${ }^{24}$ Therefore, the exchange of genetic material is something that probably occurred between membranous prebiotic precursors to life. In this context, sex amounts to a semantic concept for genetic exchange. This really refers to a biological principle which, in its various forms of equivalence in terms of transfer of information, is manifested in a patently obvious form in eukaryotes from micro-organisms to mammals, and may have arisen from primitive proto-organisms.

Numerous theories have emphasized the plausible origin of eukaryotes from an endosymbiotic process. ${ }^{25}$ It is also possible to assume that the dual nature of the genome is a consequence of parasite penetration of plas- 
mids. Some conceptions have suggested that sexual reproduction originates from primitive parasitic genetic elements.11,26,27 Among credible mechanisms for the origin of sexual reproduction, Margulis et al. ${ }^{28}$ proposed that a chimeric cell evolved via symbiogenesis by syntrophic merger between an archae and an eubacterium, and the viral eukaryogenesis theory postulates that eukaryotic cells originated from a combination of a virus, an archaea and a bacterium. ${ }^{29}$

Recently, Bazinet ${ }^{30}$ hypothesized that the sexual reproduction of eukaryotes has developed from a primitive process of parasitism with a bacterium belonging to the Rickettsia group. This hypothesis, based on the life cycle of an endosymbiotic ancestor, is exciting because these bacteria are known to influence sex determination in some animals, such as Wolbachia in woodlice. ${ }^{31,32}$ Rather than experiencing a single capture by a protoeukaryotic host, these bacteria were supposed to frequently incorporate cells over a very long period of time. In addition, the origin of mitochondria could be found in the inclusion of bacteria related to the Rickettsia group, ${ }^{33}$ and actinbased mitochondrial motility in spermiogenesis could be regarded as a vestige of the mitochondrion's rickettsial ancestry. 30

Nevertheless, there are two main objections to this hypothesis. Firstly, mitochondrial DNA has no interaction with the recombinant nuclear DNA, so mtDNA is not implicated in recombination. Secondly, Wolbachia parasitism acts by feminizing woodlice, reducing the number of males and hence restricting sexual reproduction. ${ }^{32,34}$ This feminizing process could not be associated with the development of sexual reproduction. Moreover, these assumptions do not explain why genetic exchanges generated by these parasitic relationships would provide evolutionary benefits

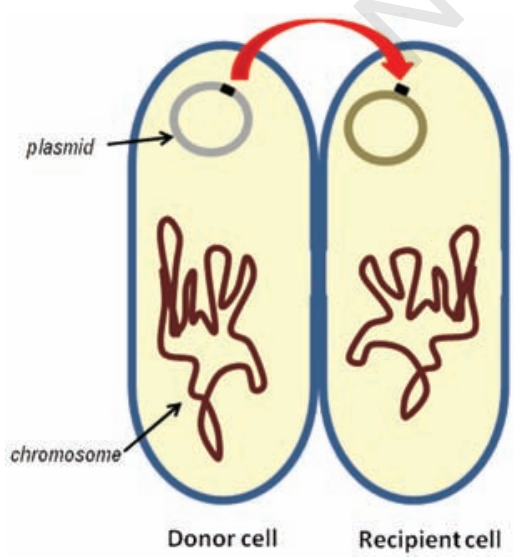

Figure 1. Genetic exchanges in bacteria. When bacteria conjugate, genetic exchanges are usually limited to plasmids, and are fragmentary. and persist in the long-term, or why the inclusion of a viral nucleus inside the proto-cell could be selected.

\section{Sex in eukaryotes}

Although sex occurs in almost all eukaryotes, in many groups sex is an optional component of reproduction. By separating sex from reproduction, the libertine bubbles theory proposed that sex originated from an archaic gene transfer process among prebiotic bubbles without the prerequisite for reproduction, ${ }^{23}$ but can this theory credibly explain differences between pro and eukaryotes?

Genetic exchanges appear to be a very primitive process, occurring in an archaic step of proto-cell formation, whereas bacteria have taken a different evolutionary path. Somehow, the eukaryotes could be derived from the most libertine bubbles, i.e. proto-cells practicing the exchange of genetic material (Figure 2). Prebiotic bubbles are microspheres which could have formed spontaneously under primitive earth conditions and which provide a crucial stage of cell development. ${ }^{35}$ Pre-biotic bubbles could offer a favourable environment for genetic material. $36-37$

The contact among the pre-biotic bubbles could, through simple food or parasitic reactions, promote the transfer of genetic material from one bubble to another. Here, a partial permeability of proto-membranes could be the key element which differentiates bacteria's peptidoglycan cell wall from eukaryotes' cellular membranes ${ }^{38}$ so that genetic exchanges could appear as a side-effect of nutritional interactions. 39

Anyway, such genetic exchanges result in an excess of genetic material in some bubbles. It has been argued that meiosis originated from a primitive DNA repair mechanism. ${ }^{40-42}$ However, DSBs double-stranded DNA breaks involved in meiotic recombination in eukaryotes have homologs in bacteria that carry out the same strand exchange functions, ${ }^{43}$ so that such a mechanism did not differ enough to make these repair mechanisms a crucial process for meiosis or to explain the hypothetical advantage of sexual reproduction. In fact, most theories have mainly focused on the evolutionary aspect of recombination, while recombination could simply be regarded as a side-effect of the excess of transferred genes.

Indeed, chromosome pairing does not appear to be a process that enhances recombination, ${ }^{44}$ and transferred DNA fragments can avoid degradation only by recombining with the genetic material of the recipient bubble. 45 Some sequences of DNA are involved in the control of gene expression while others may simply be present in the genome to act as an evolutionary buffer able to withstand nucleotide mutation without disrupting the integrity of the organism.

Consequently, it may be assumed that the nucleus has a crucial role in the neutralization of excess chromosomes inside a mass of chromatin. The binding of DNA by the histones generates a structure called the nucleosome. Heterochromatin is generally transcriptionally silent. Euchromatin, on the other hand, is more loosely packed and is where active gene transcription will be found to be taking place. Histones and other nonhistone proteins promote a fold similar to DNA in a non-chromosomal structure. The protein-DNA structure of chromatin is hence stabilized. Histone proteins are among the most highly conserved proteins in eukaryotes. The fact that histones are highly conserved molecules from a phylum to another suggests both their evolutionary importance

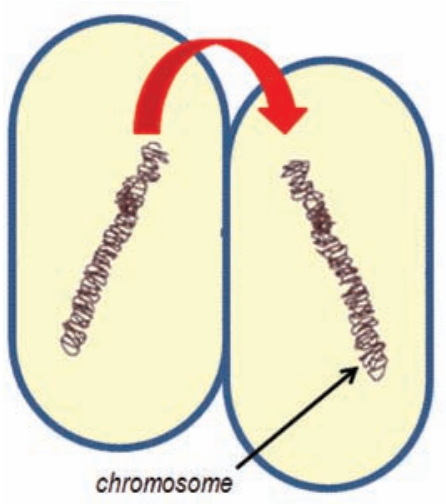

A.

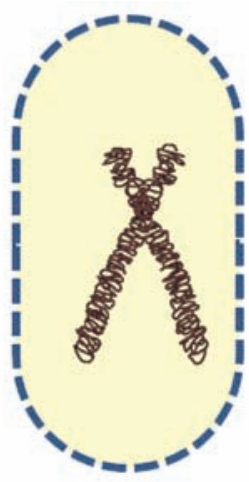

B.

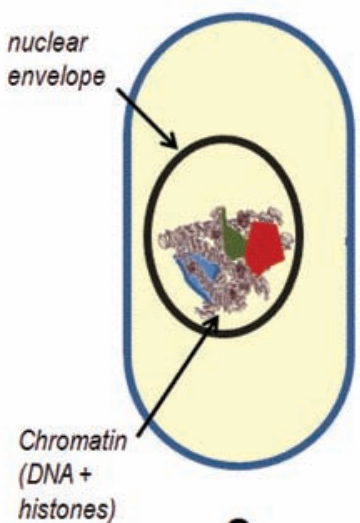

c.

Figure 2. Genetic exchanges in proto-eukaryotes: the libertine bubble theory. A) When prebiotic cells conjugate, genetic material is transmitted B) The resulting polyploidy in certain prebiotic cells could impair the proto-cell integrity $\mathrm{C}$ ) Histones, histonelike proteins and the nuclear envelope act to neutralize chromosomes in a mass of chromatin. 
and a common origin in eukaryotes. 46 The nucleosome has yet to be completed by the implementation of a nuclear membrane, including the lamina network which encloses the genetic material in this neutral form. ${ }^{47}$

Therefore, the most libertine bubbles, those which allow the transfer of genetic material, tend to interact with each other more frequently than other prebiotic-bubbles that are less prone to such interaction, providing the former with the potential to evolve. These pre-biotic bubbles then developed eukaryotic cells through a blind process of self-promoting genes correlations and compatibility. Further, these proto-eukaryotes have solved the problem of excess genetic material through a reductional process, the meiosis, resulting in the alternation of haploid-diploid stages. Meiosis has a very ancient origin ${ }^{48,49}$ and the fact that genes involved in meiosis are conserved across phyla suggests a common origin of eukaryotes. The existence of very primitive enzymes that can break the DNA molecules at meiosis has already been found, ${ }^{50}$ revealing this ancestral ability. Finally, meiosis acts as in a runaway process, as three of the four haploid genetic elements are discarded in female meiosis. By promoting unequal replication, these genes increase their own evolutionary success.

The exact nature of the origin of recombination in eukaryotic organisms is still unclear. The efficiency of pairing between homologous regions is probably dependent on both the telomere relocation and the oscillatory nuclear movements. ${ }^{48,51}$ The linear chromosomes with differentiated telomeres existed previously to the evolution of meiosis. ${ }^{45,48}$ Recombination involves exchanges between much smaller areas of DNA sequencing and requires the recognition of specific sequences by some specialized proteins.

Meiotic recombination in eukaryotic microorganisms is induced by densitydependent stressful conditions, such as overcrowding. ${ }^{24,52}$ The genetic exchanges among bubbles that lead to meiosis could be regarded as an adaptation for dealing with such environmental stress. Protozoans normally only resort to sexual reproduction when environmental conditions become adverse, because this mode of reproduction enhances the fitness of the population. ${ }^{24}$ The fact that the conjugation of protists occurs specifically under densitydependent conditions supports the hypothesis of a spontaneous exchange between bubbles leading to meiosis as an effect of overcrowding. Meiosis and genetic exchanges could hence increase the rate at which proto-cells can segregate new adaptive mutations into homozygotes, entailing a new benefit of sex. In adverse environmental circumstances, genetic exchanges could then renovate the set of proteins implied in metabolic reactions, so that interactions among libertine bubbles would be mutually advantageous. Thus, the genetic exchange seems to depend on recombination that occurs as a side effect of metabolic reactions.

\section{Eukaryotes' asexual exceptions}

Thus, the libertine bubble theory provides reasonable explanations for most of the major differences occurring between eukaryotes and bacteria i.e. diploidy, nucleosome and meiosis. However, why do some eukaryotes do not use sex?

Sex occurs before reproduction, and the reproduction does not depend on sex. Reproduction begins through a process of mitotic cell division, or embryogenesis. In fact, numerous plants, fungi, protists, polychaetes and even some vertebrates can exhibit asexual reproduction, using scissiparity or parthenogenesis. It has often been argued that sex would favour a larger genetic diversity than asexual reproduction, which produces only clones that are unable to adapt to new environmental conditions. ${ }^{53,54}$ However, genetic diversity is present in asexual populations through a variety of different clones.55,56 Some authors have pointed out the advantages of recombination, $16,57-59$ but recombination could also break up some favourable gene combinations rather than fixing advantageous correlations. ${ }^{4,60,61}$ In fact, selection cannot act as expected by numerous models because populations are finite in size and experience genetic drift. ${ }^{4}$

During their evolutionary history, the efficiency of sexual exchanges enhanced through the formation of haploid gametes. If the definition of sexes centers on the type of sexual cell being produced, there are only two sexes: males with motile sperm and females with larger eggs. Although binary mating still remains an unsolved issue, the formation of haploid gametes probably facilitated that the interaction was more efficient when restricted to two sexes. The fact that interactions between two organisms are stable enough appears to be a sufficient condition to restrict these interactions to two partners. ${ }^{6}$ Further, the evolutionary separation into two sexes could also allow avoiding lower fitness due to inbreeding, ${ }^{63}$ while an arrangement implying three or more sexes would be inefficient and biologically costly. However, the formation of a morphological and functional dissymmetry, i.e. the anisogamy, was the basis for the sexual conflict. 64 There is no reason to doubt that sexual conflict intra-locus as well as inter-locus conflict has happened throughout evolutionary history. 65 Sexual conflict leads to one sex attempting to manipulate the reproductive ability of the other. ${ }^{66}$ The disproportionate development of morphological formations underlining sexual dimorphism reveals the intensity of sexual conflict. Because one sex endeavours to manipulate the other, traits that encourage the control of the individual of the other sex (or which facilitate avoidance) are thus selected. The extreme development of intromission organs in some ducks, ${ }^{67}$ the behaviour of the female praying mantis or the toxicity of sperm in Drosophila show that the reproductive success of a sex may affect the reproduction of the other. 68

The antagonistic co-evolution generated by the sexual conflict could be one of the key events initiating the abandonment of sexual relationships. The phylogeny of ostracods or of Daphnia and related species indicates multiple transitions from diverse sexual ancestor populations to asexuality, ${ }^{69,70}$ and the intensity of sexual conflict can probably lead to the speciation of parthenogenetic populations. ${ }^{65}$ The fact that numerous parthenogenetic populations are derived from sexual species ${ }^{71}$ and that ancestors of asexual polychaetes have been found in epigamic species ${ }^{72}$ support this hypothesis well. Further, asexual organisms, from plants to animals, are often of hybrid origin associated with sexual conflict and polyploidy. ${ }^{73-75}$ The genetic divergence of parental genomes could be large enough to significantly reduce fertility in hybrids and to lead to asexual forms of reproduction.

Therefore, current sexual species could be descendants of primitive organisms that practiced more stable exchanges in the long term, while asexual species have emerged, much more recently in evolutionary history, from the conflict of interest resulting from anisogamy.

\section{Conclusions}

The biological advantage of sexual reproduction and the evolutionary maintenance of sex and meiosis have been a matter of intense debate for decades. Bacteria appear to adapt and proliferate despite the fact that they lack genes for recombination and sex. By contrast, many eukaryotes are on the verge of extinction in spite of their ability to reproduce sexually, suggesting that sex is not a good solution for reproduction. An analysis of key differences between eukaryotes and bacteria provides clear support for the libertine bubble theory which hypothesizes that sex originated from archaic prebiotic interactions - and for the hypothesis that asexual populations of eukaryotes originated from sexual species.

Focusing on primitive interactions, it is possible to suggest that selection pressures have led to the emergence of a sexual process in the primitive stages of proto-cell evolution. Rather than competition between protoeucaryotic 
bubbles, it seems reasonable to expect that self-stabilising exchanges of genetic material would, via primitive metabolism, progressively augment and enhance their efficiency. Therefore, it is interesting to emphasize the importance of bringing interactions into focus in evolutionary biology, thereby moving from genes towards an evolutionary ecology framework.

\section{References}

1. Barton NH, Charlesworth B. Why Sex and Recombination? Science 1998;281:198690.

2. Otto SP, Lenormand T. Resolving the paradox of sex and recombination. Nat Rev Genet 2002;3:252-61.

3. Kondrashov AS. Deleterious mutations and the evolution of sexual reproduction. Nature 1988;336:435-40.

4. Otto S, Gerstein A. Why have sex? The population genetics of sex and recombination. Bioch Soc Trans 2006;34:519-22.

5. West SA, Lively CM, Read A. A pluralist approach to sex and recombination. J Evol Biol 1999;12:1003-12.

6. Redfield A. Truly pluralistic view of sex and recombination. J Evol Biol 4999;12:1043-6.

7. Cooper TF, Lenski RE, Elena SF. Parasites and mutational load, an experimental test of a pluralistic theory for the evolution of sex. Proc Biol Sci 2005;272:311-7.

8. Maynard-Smith JM. The Evolution of Sex. Cambridge: Cambridge University Press 1978.

9. Bell G. The Masterpiece of Nature The Evolution and Genetics of Sexuality. Berkeley: University of California Press, 1982.

10. El Albani A. Large colonial organisms with coordinated growth in oxygenated environments 2.1 Gyr ago. Nature 2010;466: 100-4.

11. Butterfield NJ. Bangiomorpha pubescens $\mathrm{n}$ gen, $\mathrm{n}$ sp, implications for the evolution of sex, multicellularity, and the Mesoproterozoic/Neoproterozoic radiation of eukaryotes. Paleobiology 2000;26:386404.

12. Ramesh MA, Malik S, Logsdon Jr JM. A Phylogenomic Inventory of Meiotic Genes Evidence for Sex in Giardia and an Early Eukaryotic Origin of Meiosis. Curr Biol 2005; 15,185-91

13. Schubert I. 'Sex and crime' in evolution why sexualty wa so successful? Genes Genet Syst 2011;86:1-6.

14. Redfield RJ. Looking to bacteria for clues. Science 2009;325:946.

15. Otto SP. The evolutionary enigma of sex. Am Nat 2009;174Suppl 1:S1-S14
16. Christiansen FB, Otto SP, Bergman A, Feldman MW. Waiting with and without recombination: The time to production of a double mutant. Theor Pop Biol 1998;53: 199-215.

17. Cavalier-Smith T. Origins of the machinery of recombination and sex. Heredity 2002;8:125-41.

18. Cooper T.F. Recombination speeds adaptation by reducing competition between beneficial mutations in populations of Escherichia coli. PLoS Biol 2007;5:e225.

19. Lehman N. A case for the antiquity of recombination. J Mol Evol 2003;56:770-7.

20. Redfield RJ. Do bacteria have sex? Nat Rev Genet 2001;2:634-9.

21. Bendich AJ, Drlica K. Prokaryotic and eukaryotic chromosomes, what's the difference? BioEssays 2000;22:481-6.

22. Lewis PJ. Bacterial chromosome segregation. Microbiol 2001;14:519-26.

23. Lodé T. Sex is not a solution for reproduction, the libertine bubble theory. BioEssays 2011;33:419-22.

24. Bell G. Sex and Death in Protozoa. Cambridge: Cambridge University Press, 1988.

25. Margulis L. Archaeal-eubacterial mergers in the origin of Eukarya, phylogenetic classification of life. Proc Natl Acad Sci USA 1996;93:1071-6

26. Cavalier-Smith T. Cell evolution and Earth history, stasis and revolution. Phil Trans $\mathrm{R}$ Soc B Biol Sc 2006;361:969-1006.

27. Billiard S. et al. Having sex, yes, but with whom? Inferences from fungi on the evolution of anisogamy and mating types. Biol Rev 2011;86:421-42.

28. Margulis L, Dolan MF, Guerrero R. The chimeric eukaryote, Origin of the nucleus from the karyomastigont in amitochondriate protists. Proc Natl Acad Sci USA 2000;97:6954-9.

29. Bell PJ. Viral eukaryogenesis, was the ancestor of the nucleus a complex DNA virus? J Mol Evol 2001;53:251-6.

30. Bazinet C. Endosymbiotic origins of sex. BioEssays 2004;26:558-66.

31. Rousset F. et al. Wolbachia Endosymbionts Responsible for Various Alterations of Sexuality in Arthropods. Proc R Soc Lond B Biol Sc 1992;250:91-8.

32. Bouchon D, Rigaud T, Juchault P. Evidence for widespread Wolbachia infection in isopod crustaceans, molecular identification and host feminization. Proc R Soc Lond B Biol. Sc 1998;265:1081-90.

33. Andersson SGE et al. The genome sequence of Rickettsia prowazekii and the origin of mitochondria. Nature 1998;396: $133-40$.

34. Zimmer C. Wolbachia, A Tale of Sex and Survival. Science 2001;292:1093-5.

35. Fernando C, Rowe J. The origin of auto- nomous agents by natural selection. Bio Systems 2008;91:355-73.

36. Szostak JW, Bartel DP, Luisi PL. Synthesizing life. Nature 2001;409:387-90

37. Deamer D, Dworkin JP, Sandford SA, et al. The first cell membranes. Astrobiology 2002;2:371-81.

38. Mansy SS. Membrane Transport in Primitive Cells Cold Sp Harb Pers Biol 2010;2.

39. Redfield RJ. Genes for Breakfast, The Have-Your-Cake and-Eat-lt-Too of Bacterial Transformation. J Hered 1993; 84:400-4.

40. Williams G. Sex and Evolution. Princeton University Press, Princeton, 1975.

41. Bernstein H, Byerly HC., Hopf FA, Michod RE. Origin of sex. J Theor Biol 1984;110:323-51.

42. Kondrashov AS. Classification of Hypotheses on the Advantage of Amphimixis. J Hered 1993;84:372-87.

43. Egel R, Penny D. On the origin of meiosis in eukaryotic evolution, Coevolution of meiosis and mitosis from feeble beginnings. In: Sex and Recombination, Models, Means and Evolution, D. H. Lankenau, R. Egel, ed. Berlin Heidelberg: Springer; 2008. pp 249-288.

44. Wilkins AS, Holliday, R. The Evolution of Meiosis From Mitosis. Genetics 2009;181:3-12.

45. Lichten M. Meiotic recombination, Breaking the genome to save it. Curr Biol 2001;11:253-6.

46. Clarke HJ. Nuclear and chromatin composition of mammalian gametes and early embryos. Biochem Cell Biol 1992;70:85666.

47. Dahl KN, Kahn SM, Wilson KL, Discher DE. The nuclear envelope lamina network has elasticity and a compressibility limit suggestive of a molecular shock absorber. J Cell Sci 2004;117:4779-86.

48. Solari AJ. Primitive forms of meiosis, the possible evolution of meiosis. Biocell. 2002;26:1-13.

49. Extavour C. Oogenesis, Making the Mos of Meiosis. Curr Biol 2009;19:R489-91.

50. Keeney S, Giroux CN, Kleckner N. Meiosisspecific DNA double-strand breaks are catalyzed by Spo11, a member of a widely conserved protein family. Cell 1997;88:375-84

51. Scherthan H. Factors directing telomere dynamics in synaptic meiosis. Biochem Soc Trans 2006;34:550-3.

52. Bernstein H, Bernstein C. Evolutionary Origin of Recombination during Meiosis. BioScience 2010;60:498-505.

53. Ghiselin M. The economy of nature and the evolution of sex. Berkeley/Los Angeles: University of California Press, 1974.

54. Hamilton W, Zuk M. Heritable true fitness and bright birds, a role for parasites? Science 1982;218:384-7. 
55. Loxdale HD, Lushai G. Rapid changes in clonal lines, the death of a 'sacred cow. Biol J Linn Soc 2003;79:3-16.

56. Lushai G, Loxdale HD, Allen JA. The dynamic clonal genome and its adaptive potential. Biol J Linn Soc 2003;79:193-208.

57. Felsenstein J. The evolutionary advantage of recombination. Genetics 1974;78:73756.

58. Rice WR, Chippendale AK. Sexual recombination and the power of natural selection. Science 2001;294:555-9.

59. Santos M, Zintzaras E, Szathmáry E. Recombination in primeval genomes: A step forward but still along leap from maintainingasizable genome. J Mol Evol 2004;59:507-19.

60. Otto SP, Barton NH. The Evolution of Recombination, Removing the Limits to Natural Selection. Genetics 1997;147:879906.

61. Agrawal AF. Differences between selection on sex versus recombination in red queen models with diploid hosts. Evolution 2009;63:2131-41.

62. Charlesworth D, Charlesworth B. Evolutionary biology: the origins of two sexes. Curr Biol 2010;20:R519-21.

63. Czárán TL, Hoekstra RF. Evolution of sexual asymmetry. BMC Evol Biol 2004;4:34.

64 . Rice WR. Sexually antagonistic male adaptation triggered by experimental arrest of female evolution. Nature 1996;381:232-4.

65. Gavrilets S, Hayashi TI. Speciation and Sexual Conflict. Evol Ecol 2005;19:167-98.

66. Rice WR. Dangerous liaisons. Proc Natl Acad Sci USA 2000;97:12953-5.

67. McCracken KG, Wilson RE, McCracken PJ, Johnson KP. Sexual selection Are ducks impressed by drakes' display? Nature 2001;413:128.

68. Chapman T. Sexual conflict. TREE 2003;18: 41-7.

69. Bode S. et al. Exceptional cryptic diversity and multiple origins of parthenogenesis in a freshwater ostracod. Mol Phyl Evol 2010;
54:542-52.

70. Crease T, Stanton D, Hebert PDN. Polyphyletic origin of asexuality in Daphnia pulex. II. Mitochondrial DNA variation. Evolution 1989;43:1016-26.

71. Lunt D. Genetic tests of ancient asexuality in Root Knot Nematodes reveal recent hybrid origins. BMC Evol Biol 2008;8:194.

72. Nygren A, Sundberg P. Phylogeny and evolution of reproductive modes in Autolytinae Syllidae, Annelida. Mol Phyl Evol 2003;29:235-49.

73. Haig D, Wilczek A. Sexual conflict and the alternation of haploid and diploid generations. Phil Trans R Soc B Biol Sc 2006;361: $335-43$.

74. Kearney M. Hybridization, glaciation and geographical parthenogenesis. TREE 2005;20:495-502.

75. Horandl EA. Combinational theory for maintenance of sex. Heredity 2009;103: 445-57. 